\title{
Frailty and functional dependence in older population: lessons from the FREEDOM Limousin - Nouvelle Aquitaine Cohort Study
}

Sophie Boyer ${ }^{1,2,3}$, Justine Trimouillas ${ }^{2,3}$, Noëlle Cardinaud ${ }^{1,2}$, Caroline Gayot ${ }^{1,2,3}$, Cécile Laubarie-Mouret ${ }^{1,2}$, Nathalie Dumoitier ${ }^{1,4}$, Karen Rudelle ${ }^{1,4}$, Michel Druet-Cabanac ${ }^{1}$, Marie-Laure Laroche ${ }^{1,5}$ and Achille Tchalla ${ }^{1,2,3,6^{*}}$

\begin{abstract}
Background: Monitoring frailty indicators in elderly people is recommended to identify those who could benefit from disability prevention programs. To contribute to the understanding of the development of frailty in the elderly, we have created the FREEDOM-LNA cohort constituting an observational study of ageing in general population. Here, we described the characteristics of a cohort of elderly subjects who are followed for determination of frailty and loss of independence trajectories.
\end{abstract}

Results: The cohort was composed of 1085 subjects in advanced age (mean: 83.7 \pm 6.0 years) and of women in majority (68.3\%). Cardiovascular risk factors were present in $88.4 \%$ of subjects. Abnormal musculoskeletal signs were reported in $44.0 \%$ and neurologic signs in $31.9 \%$. There were $44.8 \%$ of subjects at risk of malnutrition (MNA <24) and 73.3\% (668/911) at risk of mobility-related disability (SPPB $\leq 9) ; 39 \%$ (384/973) of subjects had impaired cognitive function (MMSE< 24, adjusted on education) and 49.0\% (397/810) had signs of depression (GDS >9); 31.8\% (240/753) were frail and 58.3\% were pre-frail. Most subjects had at least one disability in ADL (66.9\%) and IADL (85.1\%). The SMAF indicated a loss of independence in 59.6\%. Overall, $59.9 \%$ of subjects could not stay at home without at least some help. Consequently, a medical consultation was proposed in 68.2 and $42.1 \%$ social supports.

Conclusions: A large part of this cohort was frail or pre-frail and presented signs of loss of independence, which may be explained by multiple factors including impaired health status, poor physical performance, cognition, isolation, depression, or nutrition. This cohort will help to determine factors that adversely influence the trajectory of physical frailty over time.

Keywords: Aging, autonomy, disability, elderly, frailty, independence

\section{Introduction}

The rise in life expectancy is one of the most remarkable advances of the last century around the world. The increased longevity is however challenged by the ageing population especially in developed countries [1, 2]. In Europe, $24 \%$ of the population is aged over 60 years

\footnotetext{
*Correspondence: achille.tchalla@unilim.fr

${ }^{6}$ Geriatric Medicine Department, University Hospital Centre, 2 Avenue Martin Luther King, 87042 Limoges, France

Full list of author information is available at the end of the article
}

and, with the post-war baby-boom generation, that proportion is projected to reach $34 \%$ in 2050. [2] Longer life promotes a progressively higher prevalence of chronic age-related comorbidities and disabling illness, including cardiovascular, metabolic, musculoskeletal, sensorial and cognitive disorders, and increasing risk of psychological distress, social disconnection, loss of independence, and dependency at the end of life $[3,4]$. In line with the geriatric community, [5-7] the World Health Organization (WHO) recently asked to adopt a global strategy to keep the elderly healthy, including providing long-term 
integrated care to maintain a level of functional ability in an age-friendly environment [4]. The objective is to keep people healthy based on the notion of functional ability and not just to treat the acute or chronic diseases $[4,5,8]$.

Pathological aging as opposed to healthy aging occurs when the organism at various organ levels is unable to compensate for age and disease-related changes [9]. On the other hand, physical and functional decline may occur in the absence of identifiable disease which has led to the concept of frailty. Frailty is defined as an agerelated state of decline and vulnerability characterized by decreased physiological reserves and function across multiple organ systems. Frail people are less resilient to sudden changes in health status even minor stressor such as mild acute illness or physical or psychological trauma, and are thus at increased risk of adverse agedrelated outcomes such as falls, hospitalizations, disability and morbi-mortality $[10,11]$. There is a considerable overlap between comorbidity, frailty and disability [12, 13]. Contrary to disability, there is current consensus that frailty is potentially reversible with appropriate interventions including physical activity, nutrition, and cognitive training in older adults. [14] Thus, monitoring frailty indicators in community-dwelling elderly people is recommended to identify old people who could benefit from disability prevention programs [15-18]. Research is also needed to determine how physical, psychologi$\mathrm{cal}$, and social conditions are associated with frailty and functional status and to determine factors that adversely influence the trajectory of physical frailty over time [19].

To contribute to the understanding of the development of frailty in the elderly, we have created the FREEDOM-LNA cohort (French acronym for Frailty, Clinical Research and Evaluation at Home in Limousin - Nouvelle Aquitaine) constituting an observatory of ageing in general population. We performed prospective and retrospective analyses of frailty, functional loss, and cognition in community-dwelling elderly with the objective to determine factors associated with frailty trajectories. A secondary objective was to analyse the different trajectories of loss of independence. In this preliminary report, we described the profile of this cohort population including health and socio-environmental factors, the loss of functional independence, and the appropriate geriatric interventions proposed to stay longer at home.

\section{Materials and methods Study design}

FREEDOM-LNA was an historical longitudinal cohort conducted by the UPSAV (University Hospital, Clinical Geriatric Department, Limoges, France). The UPSAV is a clinical unit composed of a dedicated multidisciplinary team of geriatric physicians, nurses, ergotherapists, psychomotor therapists, and social workers. The team provides global preventive geriatric assessments in general population at home with the aim to detect the risk of loss of independence and the warning signs of frailty. Subjects are solicited from various information channels including healthcare professionals (e.g. family physicians, specialists, or hospitals), social professionals, closed relatives (family members or friends), or by the subject him/ herself. The FREEDOM-LNA cohort comprised subjects aged $\geq 65$ years with at least two comorbidities, or aged $\geq 75$ years followed by the UPSAV between 01 January 2010 and 31 August 2017. All subjects were involved in a health care program that offered a comprehensive geriatric assessment every 6 months the first year and thereafter once a year. At the end of each assessment, the medical staff offered appropriate geriatric interventions including hygiene therapeutic advices, occupational therapist, psychomotor therapist, or social worker.

The study protocol was reviewed and approved by the local Institutional Review Board (CEREES, Limoges; Approval number: TPS 429,669). The protocol was also approved by the French Data Protection Authority (CNIL) insuring protection of individualized data according to the French law. Informed consent for data processing was obtained from all subjects (or legal representatives). All procedures were carried out in accordance with the 1964 Helsinki Declaration and its later amendments.

\section{Measurements \\ Demographic, socio-environmental and clinical data}

Demographic and socio-environmental characteristics were collected at inclusion and each follow-up visit. Self-reported supports including household incomes and financial supports, human supports and socio-medical supports and technical helps were also recorded using a specific questionnaire. A physical examination was performed and other clinical data, including medications, were obtained from self-reported questionnaire and from biological reports when available.

\section{Nutritional status}

The nutritional status was assessed using the Mini Nutritional Assessment (MNA). The full MNA includes 18 items grouped in 4 categories: anthropometric assessment; general assessment; short dietary assessment; and subjective assessment (self-perception of health and nutrition). Malnutrition was defined by a score $<17$ and a risk of malnutrition by a score between 17 and 23.5 [20].

\section{Physical activity and mobility}

Mobility was assessed using the Short Physical Performance Battery (SPPB) which consists of a 4-meter walk 
at usual pace, a timed repeated chair stand, and three increasingly more difficult standing balance tests [21]. The total score ranges from 0 (worst) to 12 (best). A SPPB score $\leq 9$ was suggesting for a risk of mobility-related disability.

\section{Frailty}

Frailty was assessed using the five phenotypic criteria as described by Fried et al. [10]: weakness as measured by grip strength (dominant hand $<20 \%$ ), slowness (walking speed $<20 \%$ of normal), low level of physical activity in the last 2 weeks $(<20 \%$ of energy expenditure, based on a physical activity questionnaire), low energy or selfreported exhaustion, and unintentional weight loss ( 4 to $5 \mathrm{~kg}$ since the previous year). Subjects were considered as frail when at least 3 criteria were present, pre-frail when there was one or two criteria and robust when there was no criteria.

\section{Health status}

The health status was assessed using the EuroQol-5 Dimension (EQ-5D). Each item of five dimensions (mobility, self-care, usual activities, pain/discomfort, and anxiety/depression) was scored using a 3-point scale (no problem $=1$, with problems $=2$; with extreme problems=3). The subjects were also asked to value their own health status on an analogue scale (EQ-VAS) ranging from 0 (the worst possible health status) to 100 (the best possible health status) [22].

\section{Cognitive and psychosocial status}

Neurocognitive domains such as verbal memory, immediate memory, and executive functioning were assessed using various neuropsychological tests including the Mini Mental State Examination (MMSE) questionnaire (30 items, scored between 0 and 30), [23] the 5-word test (5WT), [24] the clock drawing test (CDT), [25] the Controlled Word Association Test, [26] and the Category Naming Test [27]. Subjects were considered to have a cognitive deficit if MMSE was $\leq 20$ in subjects with low education, $\leq 23$ in subjects with medium education and $\leq 26$ in subjects with a high education. A poor memory performance was indicated by 5 -WT score $\leq 9$. Depression over the past week was monitored using the Geriatric Depression Scale (GDS, 30 items); scores ranging from 0 to 5 indicate normal mood; scores between 5 and 9 indicate a risk of depressive symptoms, and scores $>9$ indicate severe depressive symptoms [28].

\section{Functional status}

The functional status was assessed using the Katz's index for basic daily living (ADL), and using the Lawton's scale for instrumental activities of daily living
(IADL) [29, 30]. An ADL $\leq 5$ indicates dependency for daily activity, and an IADL $\leq 7$ dependency for instrumental daily activities. Independence was also assessed using the SMAF (French acronym for Functional Autonomy Measurement System) questionnaire [31]. The SMAF is a 29-item scale and measures functional ability in 5 areas: daily living activities (7 items), mobility (6 items), communication (3 items), mental functions (5 items) and domestic tasks (8 items). For each item, the disability was scored on a 5-point scale: 0 (independent), -0.5 (with difficulty), -1 (needs supervision), -2 (needs help), and -3 (dependent). The total scored from 0 to -87 and a score $\leq-16$ was subjective of a loss of independence. The level of dependency was also assessed using the actual legal instrument for evaluating dependency in elderly in France (AGGIR) [32] This is a 17 -item questionnaire which covered relatively complex activities related to physical or domestic functions (walking, dressing, toileting, household cleaning ...), cognitive or social functions (cooking, medication use, finances, leisure, etc.). Each activity is scored according to three levels of dependency. This leads to calculate 3 degrees of dependency: strong dependency (GIR1 or 2), moderate dependency (GIR3 and 4) and weak dependency (GIR 5 or 6).

\section{Geriatric intervention}

Geriatric interventions such as therapeutic-hygienic and preventive advices; treatment modifications; additional medical and social assessments; reeducation/ readaptation in an occupational therapist; psychosocial readaptation in a psychotherapist were proposed at the end of each visit according to the subject's need.

\section{Statistical analyses}

For subjects included between 2010 and 31 January 2014, data were recorded on the subject's file and then entered in the software dedicated to the study. For subjects included between 01 and 2014 and 31 August 2017 , data were directly entered in the software system. Statistical analyses were performed using the SAS software, version 9.4 (SAS Institute, Cary, NC, USA). The statistical analysis focused on subjects characteristics at first visit (inclusion). Quantitative variables were described using means, standard deviations (SD), medians, quartiles, minimal, and maximal values and qualitative data were described using number of cases and percentages. Missing data were not replaced, and percentages were calculated without accounting for missing data, unless otherwise specified. 


\section{Results}

Overall, 1337 subjects were included; 250 (18.7\%) had no data recorded, and 2 subjects refused their data to be analysed. Thus, the analysed population was composed of 1085 subjects. Main subjects' characteristics are provided in Table 1 . The cohort was mainly composed of elderly subjects of 80 years old or above (73.6\% of subjects) and of women in majority (68.3\%), with a low/medium educational level (61.9\%), and living alone (53.8\%). Most subjects $(88.5 \%)$ had at least one cardiovascular risk factor and are exposed to polypharmacy, $83.4 \%$ taking 5 and more medications daily. Clinical examination showed abnormal cardiovascular signs in $82.2 \%$ of subjects, musculoskeletal signs in $44.0 \%$, and neurologic signs in $31.9 \%$ of subjects.

\section{Nutritional status, physical activities and frailty}

Overall, the mean MNA score was $23.3 \pm 4.1$ and a risk of malnutrition (MNA < 24) was shown in 44.8\% (586/1062) of subjects. The mean SPPB total score was $6.7 \pm 3.6$, and $73.3 \%(668 / 911)$ subjects had a SPPB $\leq 9$, thus a risk of mobility-related disability.

The frailty total score was determined in 754 subjects; $240(31.8 \%)$ were frail, $439(58.2 \%)$ were pre-frail and 75 $(9.9 \%)$ were robust. The most frequent frailty phenotype was "weakness" in 771/934 (82.5\%) subjects and "low level of physical activity" in 587/969 (58.7\%) subjects, while "slowness" was found in 262/792 (33.1\%) subjects, "exhaustion" in 211/929 (22.7\%) subjects, and "weight loss" in 106/1070 (9.9\%) subjects.

\section{Mental functions}

Thirty-nine percent (384/974) of subjects had a cognitive deficit (low MMSE adjusting for education); 20.6\% $(170 / 826)$ had a 5 -WT $<9$ indicating poor memory performances; $50.7 \%$ (417/823) failed on the clock-drawing test indicating some executive dysfunction; and $70.1 \%$ $(510 / 727)$ had at least one categorial or literal fluency considered as pathologic, thus suspecting cognitive impairment. Regarding the depression scale, 33.6\% $(272 / 810)$ had a GDS score between 5 and 9 indicating a risk of depression and 49.0\% (397/810) had a GDS score > 9 , clearly indicating a depression.

\section{Functional status}

The mean EQ-VAS was $57.8 \pm 18.2$ indicating impaired health status on average. The most affected dimension was pain/discomfort (88.8\% including $9.3 \%$ with extreme pain/discomfort). Anxiety/depression was the second most affected dimension (67.2\% reported moderate or extreme anxiety/depression). Using this
Table 1 Socio-demographic and clinical characteristics

\begin{tabular}{|c|c|}
\hline Age, mean $\pm S D,(N=1071)$ & $83.7 \pm 6.0$ \\
\hline Sex, women, $\mathrm{n} / \mathrm{N}(\%)$ & $740 / 1085(68.3 \%)$ \\
\hline \multicolumn{2}{|l|}{ Marital status and children $(N=1083)$} \\
\hline Married/common low partner & $467(43.1 \%)$ \\
\hline Widowed/divorced/unmarried & $616(56.9 \%)$ \\
\hline At least one children & $957(88.4 \%)$ \\
\hline Living alone & $583(53.8 \%)$ \\
\hline \multicolumn{2}{|l|}{ Socio-professional category ${ }^{\mathbf{a}}(N=1031)$} \\
\hline Low & $736(71.4 \%)$ \\
\hline Intermediate & $180(17.5 \%)$ \\
\hline High & $115(11.2 \%)$ \\
\hline \multicolumn{2}{|l|}{ Education $^{\mathbf{b}}(N=1083)$} \\
\hline Low & $203(18.7 \%)$ \\
\hline Medium & $468(43.2 \%)$ \\
\hline High & $412(38.0 \%)$ \\
\hline \multicolumn{2}{|l|}{ Vital signs (mean $\pm S D$ ) } \\
\hline Systolic blood pressure (mmHg), N=1039 & $134.3 \pm 16.8$ \\
\hline Diastolic blood pressure $(\mathrm{mmHg}), N=1039$ & $73.3 \pm 10.0$ \\
\hline Heart rate (bpm), $N=1018$ & $70.9 \pm 9.7$ \\
\hline Body mass index $\left(\mathrm{kg} / \mathrm{m}^{2}\right), N=1054$ & $26.5 \pm 5.2$ \\
\hline \multicolumn{2}{|l|}{ Abnormal clinical examination $(N=1074)$} \\
\hline Cardiovascular & $882(82.1 \%)$ \\
\hline Musculoskeletal system & $473(44.0 \%)$ \\
\hline Neurologic & $343(31.9 \%)$ \\
\hline Skin & $170(15.8 \%)$ \\
\hline Abdomen & $140(13.0 \%)$ \\
\hline Oral health & $134(12.5 \%)$ \\
\hline Pulmonary & $100(9.3 \%)$ \\
\hline Hydration & $51(4.7 \%)$ \\
\hline \multicolumn{2}{|l|}{ Cardiovascular morbidities, $\mathrm{n} / \mathrm{N}(\%)$} \\
\hline Hypertension & 792/1054 (75.1\%) \\
\hline Dyslipidaemia & $497 / 1049(47.4 \%)$ \\
\hline Obesity & $272 / 1047(26.0 \%)$ \\
\hline Diabetes & $226 / 1048(21.6 \%)$ \\
\hline Smoking & $140 / 1045(13.4 \%)$ \\
\hline Alcohol consumption & $47 / 1050(4.5 \%)$ \\
\hline At least one comorbidity & $933 / 1055(88.4 \%)$ \\
\hline At least two comorbidities & $605 / 1055(57.3 \%)$ \\
\hline 5 and more medications daily & $846 / 1014(83.4 \%)$ \\
\hline \multicolumn{2}{|l|}{ Health status and frailty } \\
\hline MNA total score, mean $\pm S D,(N=1062)$ & $23.3 \pm 4.1$ \\
\hline$<17$ & $80 / 1062(7.5 \%)$ \\
\hline $17-24$ & $396 / 1062(37.3 \%)$ \\
\hline$\geq 24$ & $586 / 1062(55.2 \%)$ \\
\hline SPPB total score, mean $\pm S D$, $(N=911)$ & $6.7 \pm 3.6$ \\
\hline $0-6$ & $428 / 911(47.0 \%)$ \\
\hline $7-9$ & 240/911 (26.3\%) \\
\hline $10-12$ & 243/911 (26.7\%) \\
\hline Fried total score, mean $\pm S D,(N=753)$ & $2.0 \pm 1.2$ \\
\hline Frail & 240/753 (31.9\%) \\
\hline Pre-frail & $439 / 753(58.3 \%)$ \\
\hline
\end{tabular}


Table 1 (continued)

\begin{tabular}{|c|c|}
\hline Robust & $74 / 753(9.8 \%)$ \\
\hline EQ-VAS, mean $\pm S D$, $(N=792)$ & $57.8 \pm 18.2$ \\
\hline \multicolumn{2}{|l|}{ Mental function } \\
\hline MMSE total score, mean \pm SD, $(N=973)$ & $24.2 \pm 4.9$ \\
\hline Pathologic MMSE & $384 / 973(39.5 \%)$ \\
\hline $5-\mathrm{WT}$, mean $\pm \mathrm{SD},(N=826)$ & $9.1 \pm 1.7$ \\
\hline $5-W T<9$ & $170 / 826(20.6 \%)$ \\
\hline CDT failure & $417 / 823(50.7 \%)$ \\
\hline At least one pathologic verbal fluency & $510 / 727(70.2 \%)$ \\
\hline Pathologic categorial fluency & $451 / 727(62.0 \%)$ \\
\hline Pathologic literal fluency & $227 / 727(31.2 \%)$ \\
\hline$G D S$, mean $\pm S D,(N=810)$ & $9.9 \pm 5.5$ \\
\hline $5-9$ (risk of depression) & $272 / 810(33.6 \%)$ \\
\hline 9 (depression) & $397 / 810(49.0 \%)$ \\
\hline \multicolumn{2}{|l|}{ Functional status } \\
\hline ADL score, mean $\pm S D,(N=1070)$ & $5.0 \pm 1.2$ \\
\hline $\mathrm{ADL} \leq 5$ & $716 / 1070(66.9 \%)$ \\
\hline IADL score, mean $\pm S D,(N=1069)$ & $5.1 \pm 2.3$ \\
\hline $\mid A D L \leq 7$ & $910 / 1069(85.1 \%)$ \\
\hline SMAF total score, mean $\pm \mathrm{SD},(N=1063)$ & $-21.6 \pm 14.5$ \\
\hline $0--8$ (independent) & $197 / 1063(18.5 \%)$ \\
\hline$-8--16$ (moderate dependency) & $232 / 1063(21.8 \%)$ \\
\hline$\leq-16$ (loss of independence) & $634 / 1063(59.6 \%)$ \\
\hline GIR score, mean $\pm S D$, $(N=1053)$ & $4.6 \pm 1.3$ \\
\hline GIR 1 or 2 (strong dependency) & $66 / 1053(6.3 \%)$ \\
\hline GIR 3 or 4 (moderate dependency) & $474 / 1053(45.0 \%)$ \\
\hline GIR 5 or 6 (mild dependency) & $513 / 1053(48.7 \%)$ \\
\hline
\end{tabular}

a Low (employee, worker, farmer, housewife/husband); High (Manager, Executive manager, licensed professional), intermediate (other status)

${ }^{\mathrm{b}}$ Low (can read, write, count); medium (primary certificate level); high (secondary school, high school, university)

questionnaire, $61.8 \%$ reported having difficulties in doing usual activities, $52.5 \%$ in washing or dressing alone, and $57.2 \%$ in walking (See Supplementary Table S1).

The mean GIR score was $4.6 \pm 1.3$ indicating that on average the cohort population had some dependency. Overall, $66.9 \%(716 / 1070)$ of subjects were dependent in ADL and $85.1 \%(910 / 1069)$ in IADL, and 59.6\% $(637 / 1063)$ had a SMAF $\leq-16$ indicating a loss of independence. As shown in Fig. 1, the most affected SMAF dimension was "Instrumental activities of daily living" (mean score per item $-1.28 \pm 0.84$ ) and then "Daily activities" $(-0.70 \pm 0.56)$. Beside difficulties in "Instrumental activities of daily living" (i.e. cleaning, cooking, shopping, laundry), the most severely affected activities was "grooming" $(-1.49 \pm 0.88)$; "using the stairs" $(-0.91 \pm 0.97)$, "walking outside" $(-0.83 \pm 0.89)$, memory $(-0.93 \pm 0.68)$ and judgement (-0.83 \pm 0.96$)$ (Fig. 2).
Socio-environmental conditions and supports

A description of the main available supports including financial, human and technical is provided in Table 2 . Most subjects $(77.8 \%)$ were owner or had a free of charge lodging. The household income was quite low $(<1500$ $€$ per month) in $46.7 \%$ (485/1038). Almost all (97.2\%) were covered by a private health insurance, and $73.1 \%$ for long-lasting illness. In addition, 31.3\% received a personalised allowance of autonomy (i.e. monthly amount of $138 \pm 243 €)$. Overall $89.8 \%$ of subjects could rely on human support including relatives (64.0\%), nurses or home care nurse services (63.3\%) and domestic help (52.9\%). Regarding technical support, 83.8\% used at least a technical help, mainly an alarm system (61.3\%), grab bars $(45.6 \%)$, or sticks $(43.1 \%)$.

Overall, 68.0\% wereliving in a house. Most dwellings appeared not fully adapted including presenceof onside or outside stairs ( $78.2 \%$ and $58.7 \%$, respectively); $50.2 \%$ had a showerand $39 \%$ a bathtub but in most cases, they were not adapted, and/or notaccessible(See Supplementary Table S2).

\section{Geriatric interventions}

At the end of the assessment,it was considered that $381 / 1063(35.8 \%)$ subjects could stay at home withoutdifficulties, while 637 (59.9\%) subjects required some help. Staying at homewas considered as only possible in condition of human support in $681(64.0 \%)$ subjects, technical support in 503 (47.3\%) subjects, or financial support in $409(38.5 \%)$ subjects. Institutionalisation was requested for 59 (5.5\%) subjects andshould be considered in 149 (14.0\%) other subjects. A description of proposed interventionsfollowing the geriatric assessment is provided in Table 3. This included preventive therapeutic andnutritional advices ( $78.2 \%$ of subjects), a medical consultation (68.2\%), anevaluation by a social worker (42.1\%), or a psychomotor therapist (42.1\%), and therapeuticmodification $(39.8 \%)$.

\section{Discussion}

The goal of this preliminary report was to determine the profile of the FREEDOM-LNA cohort. The cohort included 1085 community-dwelling elderly subjects, in advanced age (83years on average) and composed of women in majority. More than half of subjects were living alone, had several cardiovascular risk factors and are exposed to polypharmacy. Overall, they presented with a very low physical capacity and mobility-related disability and $30-50 \%$ showed significant cognitive deficit, depression and a risk of malnutrition. Most subjects were frail or prefrail and in loss of independence and thus required help to stay at home. Overall, the health status based on 


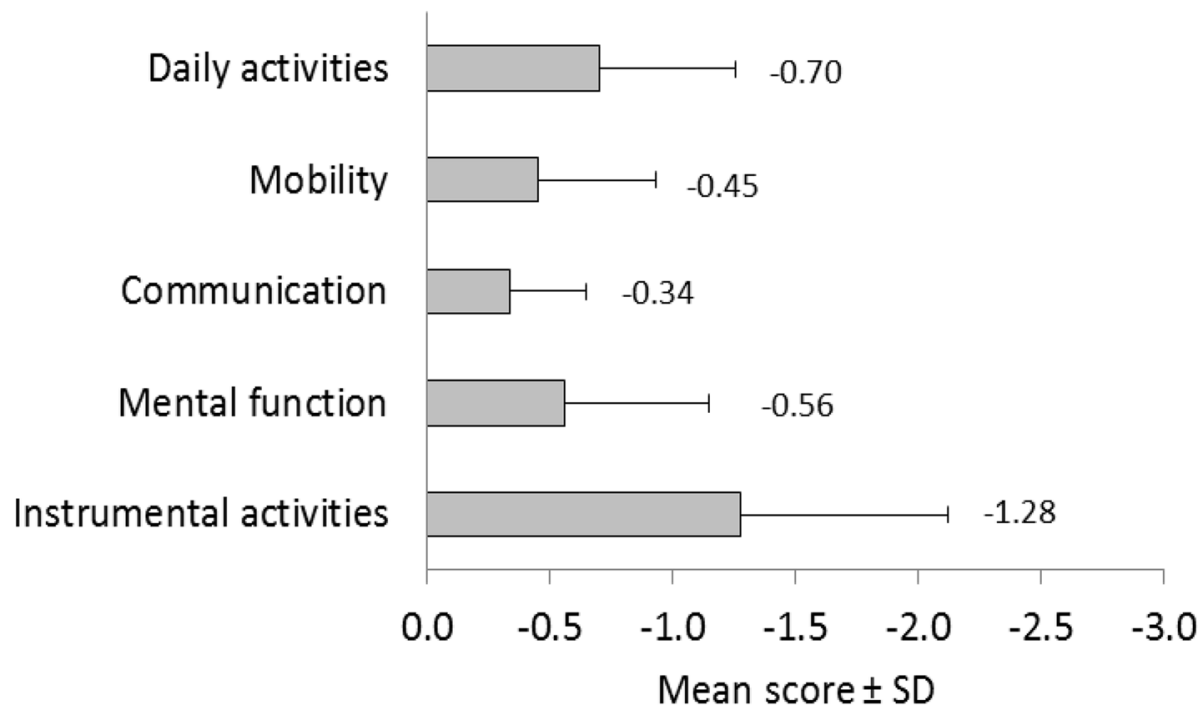

Fig. 1 SMAF item for each domain. The mean \pm SD score of each item of the 5 SMAF domains are indicated. A score of -1 indicates that subjects need some help to perform the function or activity, a score of -2 indicates a difficulty to perform the task, and a score of -3 the impossibility of doing the task

\section{A: Daily activities}

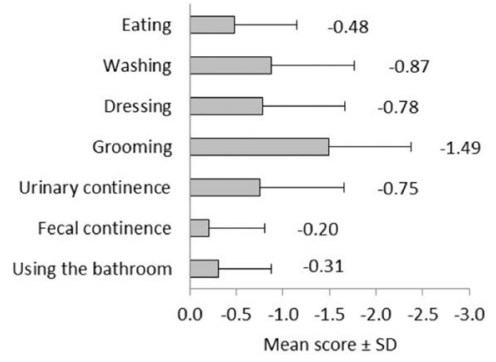

D: Mental function

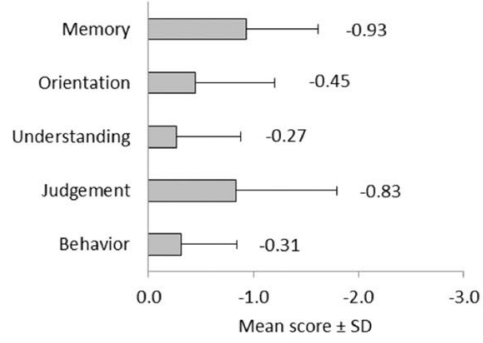

\section{B: Mobility}

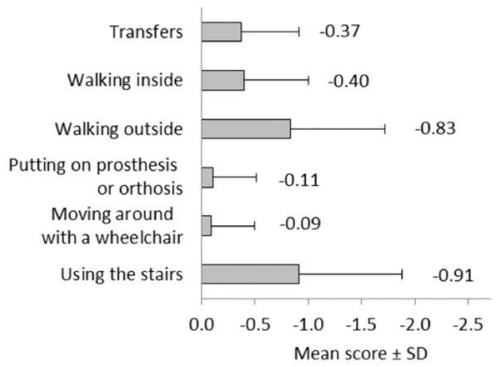

\section{E: Instrumental activities of daily living}

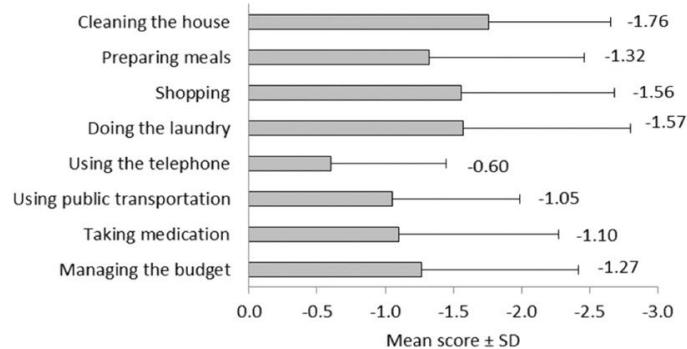

Fig. 2 Score of each SMAF item. A Daily activities; B Mobility; C Communication; D Mental function; E Instrumental activities of daily living

the EQ-5D questionnaire was worse compared to another cross-sectional study in advanced elderly, [33] with a substantial proportion of subjects reporting pain/discomfort, mobility difficulties, and anxiety/depression. The overall health status of the FREEDOM-LNA population was also worse compared to a cohort of communitydwelling older subjects selected for their ability to walk 20 feet without personal assistance, [34] but quite better compared to another small clinical trial in our clinical centre with frail elderly people, [35] and compared to 
Table 2 Resources and supports

\begin{tabular}{|c|c|c|}
\hline \multirow[t]{4}{*}{ Lodging } & N & 1083 \\
\hline & Owner & $646(59.6 \%)$ \\
\hline & Tenant & $241(22.3 \%)$ \\
\hline & Usufruct/free of charge lodging & $196(18.1 \%)$ \\
\hline \multirow{5}{*}{$\begin{array}{l}\text { Monthly house- } \\
\text { hold income } \\
\text { (Euros) }\end{array}$} & $\mathrm{N}$ & 1038 \\
\hline & $<1000$ & $183(17.6 \%)$ \\
\hline & $1000-1500$ & $302(29.1 \%)$ \\
\hline & $1500-2000$ & $241(23.2 \%)$ \\
\hline & $>2000$ & $312(30.1 \%)$ \\
\hline \multirow[t]{3}{*}{ Financial support } & Private health insurance & 1013/1042 (97.2\%) \\
\hline & Coverage for long-lasting illness & 769/1052 (73.1\%) \\
\hline & $\begin{array}{l}\text { Personalised allowance of } \\
\text { autonomy }\end{array}$ & $325 / 1040(31.3 \%)$ \\
\hline \multirow[t]{10}{*}{ Human support } & At least one human support & 973/1085 (89.8\%) \\
\hline & Relatives & $582 / 910(64.0 \%)$ \\
\hline & Domestic help & $502 / 949(52.9 \%)$ \\
\hline & Nurse & $506 / 933(54.2 \%)$ \\
\hline & Home care nurse service & $78 / 854(9.1 \%)$ \\
\hline & Physiotherapist & $271 / 882(30.7 \%)$ \\
\hline & Meals-on-wheels & $222 / 881(25.2 \%)$ \\
\hline & Housekeeper & 153/871 (17.6\%) \\
\hline & Assistant social worker & $106 / 853(12.4 \%)$ \\
\hline & Other & 197/789 (25.0\%) \\
\hline \multirow[t]{13}{*}{ Technical support } & N & 1083 \\
\hline & At least one technical help & $908(83.8 \%)$ \\
\hline & Alarm system & $664(61.3 \%)$ \\
\hline & Stick & $467(43.1 \%)$ \\
\hline & Crutch & $138(12.7 \%)$ \\
\hline & Walker & $197(18.2 \%)$ \\
\hline & Grab bar & $494(45.6 \%)$ \\
\hline & Chair wardrobe & $173(16.0 \%)$ \\
\hline & Booster seat & $161(14.9 \%)$ \\
\hline & Wheelchair & $74(6.8 \%)$ \\
\hline & Medical bed & 107 (9.9\%) \\
\hline & Lifting apparatus & $4(0.4 \%)$ \\
\hline & Other & 455 (42.0\%) \\
\hline
\end{tabular}

Table 3 Geriatricinterventions proposed at the end of first visit

\begin{tabular}{ll}
\hline Medical plan & $740(68.2 \%)$ \\
Preventive hygieno-therapeutic advices & $848(78.2 \%)$ \\
Consultation (family physician, specialist) & $741(68.2 \%)$ \\
Evaluation by a social worker & $457(42.1 \%)$ \\
Evaluation by a psychomotor therapist & $457(42.1 \%)$ \\
Therapeutic modification & $432(39.8 \%)$ \\
Evaluation by an occupational therapist & $328(30.2 \%)$ \\
Complementary exam & $170(15.7 \%)$ \\
Scheduled hospitalisation & $125(11.5 \%)$
\end{tabular}

older adults admitted to our emergency geriatric medicine unit [36].

Disability in essential daily activities is considered as an adverse outcome of frailty. In this study, $32 \%$ of subject were frail based on the Fried's criteria and another considerable proportion (58\%) was pre-frail. In a systematic review of the literature, the reported prevalence of frailty in elderly among the community worldwide was variable ranging between $4 \%$ and $59 \%$ and the meta-analysis showed a weighted prevalence of $10.7 \%$, [37] which is quite lower compared to the rate in the FREEDOM-LNA study. In another literature review, Shamliyan et al. estimated the prevalence of the frail phenotype to $26 \%$ in people over 85 years [38]. In another cross sectional study in France, physical frailty was reported in $9.5 \%$ of people aged $70-79$ years, $18.4 \%$ of people $80-89$ years, and $25.3 \%$ of people aged $\geq 90$ years [39]. In our study, the most frequent frailty criteria were weakness and low activity. These two frailty criteria have been shown to be the most powerful predictors of ADL disability [40]. In this study, it is noteworthy that the rate of subjects with some disabilities was substantially higher (66.9\% had difficulties in at least one ADL, and $85.2 \%$ in at least one IADL) than the rate of frail subjects. By comparison, in another French cross-sectional study, 15.0 and $22.4 \%$ of elderly of similar age had difficulties in at least one ADL and IADL, respectively [41].

Loss of independence in our cohort was consistent for more than $51.3 \%$ of subjects as indicated by the GIR scores, and $59.5 \%$ of subjects using SMAF. They predominantly needed help to do most executive functions and for "grooming". Other daily activities were performed with difficulties, and some may be limited to alteration in mobility ("walking outside", or "using stairs) or mental function ("memory", "judgement"). Overall, the disability profile is consistent with an early phase of loss of independence [42]. Possible causes of loss of independence included musculoskeletal and neurological disorders which were reported by 56 and 32\% of subjects, respectively, and also cognitive or mental decline in $30-50 \%$ of subjects. Our results also showed that almost $75 \%$ had a risk of mobility-related disability as assessed using the SPPB questionnaire. A low SPPB score has been previously associated with an increased risk of frailty, disability in daily life activities, falling, hospitalisation, and nursing home admission. [43, 44]. Moreover, social isolation and depression can also lead to frailty and decline in functional status [45]. In this cohort, more than half of the population were living alone and $49 \%$ had signs of depression. On the other hand, the home environment can also influence the ability to perform ADL, and we found that it was frequently not adapted with stairs and inaccessible showers or bathtubs. 
Nutrition is believed to influence age-related frailty, cognition and disability, and adverse health outcome [46, 47]. Here, we used the MNA questionnaire which can be considered as a valuable tool to identify frail elderly subjects at risk of malnutrition, especially because it encompasses physical and mental aspect of health including mobility, psychological stress or acute disease in the previous 3 months [20]. It can also predict the risk of malnutrition when serum albumin and BMI are still normal, which was the case in the FREEDOM-LNA cohort. Here, we found that $7.5 \%$ of subjects were clearly malnourished (MNA < 17) which seems low compared to the rate of frailty subjects and compared to another small clinical trial in frail older subjects referred to our clinical centre [35]. Nevertheless, a high proportion (37\%) of subjects is considered at risk of malnutrition.

Taken together, the baseline characteristics of the FREEDOM-LNA cohort showed a heterogeneous population of elderly particularly aged, frail or prefrail and presenting with frequent multimorbidity, and at risk of loss of independence due to low physical capacity and alteration of cognition. At the end of this geriatric assessment, it was considered that most subjects needed human support to be able to stay at home. Technical and financial conditions may be an issue, thus requiring intervention. The independent factors associated with frailty, functional loss and cognition will be analysed in an upcoming report.

As observational, our cohort has some limitations, mainly due to selective and information biases. First, the cohort was composed of community-dwelling subjects who were interested to receive a comprehensive geriatric assessment at home. Thus, such assessment may be less considered in apparently healthy elderly subjects. In addition, it is not known exactly if the subjects were addressed for primary or secondary prevention. According to an estimation between 2010 and 2017, interventions by our clinical centre were mainly solicited by the subject or a relative (45.5\%), followed by hospital (30.8\%), familial physicians $(15.4 \%)$ or others (7.9\%) (Personal data, not published). Nevertheless, our aim was not to obtain a representative sample of the general population, but rather to constitute an observatory of elderly subjects at risk of loss of independence. Next, we used the frailty criteria defined by Fried et al [10]. This is the most frequent screening tool used for frailty and was shown to be independently predictive of incident falls, worsening mobility or ADL disability, hospitalization, and death in the elderly. However, this restricts the multidomain of frailty to a physical phenotype, and thus do not completely consider the impact of cognitive and emotional function in development and progression of frailty [48]. Nevertheless, various neuropsychological tests were used in our study to measure cognitive and depressive functions and their relationship with disability and frailty. This will be analysed in separate reports. Finally, some percentages may be overestimated due to missing data including cognitive tests (i.e. GDS, CDT, verbal fluency) and frailty.

In conclusion, the FREEDOM-LNA cohort is composed of advanced elderly with various risk factors of frailty and disability associated with low health status, and impaired physical and cognitive functions. This cohort will help to determine factors that adversely influence the trajectory of physical frailty over time.

\section{Abbreviations \\ ADL: Activity of Daily Living; AGGIR: Instrument for evaluating dependency in elderly in France; CGA: Comprehensive Geriatric Assessment; EQ-5D: EuroQol-5 Dimension; FRIED'S CRITERIA: Physical frailty; FREEDOM-LNA cohort: French acronym for Frailty, Clinical Research and Evaluation at Home in Limou- sin - Nouvelle Aquitaine; GDS: Geriatric Depression Scale; IADL: Instrumental Activities for Daily Living; MMSE: Mini Mental State Examination; MNA: Mini Nutritional Assessment; SMAF: Functional Autonomy Measurement system; SPPB: Short Physical Performance Battery; UPSAV: Geriatric mobile team (Unité de Prévention de Suivi et d'Analyse du Vieillissement).}

\section{Supplementary Information}

The online version contains supplementary material available at https://doi. org/10.1186/s12877-022-02834-w.

Additional file 1: Table S1. EQ-5Dquestionnaire. Table S2. Habitation

and equipment.

\section{Acknowledgements}

The authors would like to thank study participants, Axonal-Biostatem (Castries, France) for data management and statistics, and Thierry Radeau Consulting (Epinay-Sous-Senart, France), the University of Limoges Partenariale Foundation and "Carsat Sud Ouest" de Limoges.

\section{Authors' contributions}

AT and SB drafted the manuscript. AT, ND, KR, MDC and MLL read and revised the manuscript. CB helped perform statistical analysis. AT, NC, CG, CLM, JT collected data. AT, SB and JT participated in the design of the study methodology and helped draft the manuscript. All authors read and approved the final manuscript.

\section{Funding}

No funding was obtained for this study.

\section{Availability of data and materials}

Doctor Sophie Boyer, PhD (sophie.boyer@chu-limoges.fr) who should be contacted if someone wants to request the data. Data are not publicly available due to privacy or ethical restrictions.

\section{Declarations}

Ethics approval and consent to participate

The study protocol was reviewed and approved by the local Institutional Review Board (CEREES, Limoges; Approval number: TPS 429669). The protocol was also approved by the French Data Protection Authority (CNIL) insuring protection of individualized data according to the French law. Informed consent for data processing was obtained from all subjects (or legal representatives). All procedures were carried out in accordance with the 1964 Helsinki Declaration and its later amendments. 


\section{Consent to publish \\ Not Applicable.}

\section{Competing interests}

The authors declare no competing interests.

\begin{abstract}
Author details
${ }^{1}$ Laboratoire VieSanté - UR 24134 (Vieillissement, Fragilité, Prévention, e-Santé), Institut $\Omega$-Health, Université de Limoges, Limoges, France. ${ }^{2}$ UPSAV (Unité de Prévention, de Suivi et d'Analyse du Vieillissement), Pôle HU Gérontologie Clinique, CHU de Limoges, 2 Avenue Martin-Luther, F-87042 Limoges, King, France. ${ }^{3}$ Unité de Recherche Clinique et d'Innovation (URCI) de Gérontologie, Pôle HU Gérontologie Clinique, CHU de Limoges, Limoges, France. ${ }^{4}$ Département de Médecine Générale, Faculté de Médecine de Limoges, Limoges, France. ${ }^{5}$ Centre de Pharmacovigilance et de Pharmacoépidémiologie, CHU de Limoges, Limoges, France. ${ }^{6}$ Geriatric Medicine Department, University Hospital Centre, 2 Avenue Martin Luther King, 87042 Limoges, France.
\end{abstract}

Received: 20 July 2021 Accepted: 8 February 2022

Published online: 14 February 2022

\section{References}

1. European Commission. The 2015 Ageing Report. Underlying Assumptions and Projection Methodologies. Brussels: European Commission. European Union; 2014. Available from: https://ec.europa.eu/economy_ finance/publications/european_economy/2014/pdf/ee8_en.pdf (Acces sed on 30 November 2020).

2. United Nations, Department of Economic and Social Affairs, Population Division World Population Prospects: The 2015 Revision, Key Findings and Advance tables Working Paper No. ESA/P/WP.241). New York: United Nations; 2015. 66 p. Available from: https://esa.un.org/unpd/wpp/publi cations/files/key_findings_wpp_2015.pdf (Accessed on 30 Nov 2020).

3. Kojima G, Liljas AEM, lliffe S. Frailty syndrome: implications and challenges for health care policy. Risk Manag Healthc Policy 2019; 12:23-30.

4. Beard JR, Officer A, de Carvalho IA, et al. The World report on ageing and health: a policy framework for healthy ageing. Lancet 2016:387(10033):2145-2154

5. Friedman SM, Shah K, Hall WJ. Failing to Focus on Healthy Aging: A Frailty of Our Discipline? J Am Geriatr Soc 2015;63(7):1459-1462.

6. Friedman $\mathrm{SM}$, Mulhausen $\mathrm{P}$, Cleveland ML, et al. Healthy Aging: American Geriatrics Society White Paper Executive Summary. J Am Geriatr Soc 2019;67(1):17-20.

7. Rolfson D. Successful Aging and Frailty: A Systematic Review. Geriatrics (Basel). 2018;3(4):79.

8. Crimmins EM. Lifespan and Healthspan: Past, Present, and Promise. Gerontologist 2015;55(6):901-911.

9. Fulop T, Larbi A, Witkowski JM, et al. Aging, frailty and age-related diseases. Biogerontology. 2010;11(5):547-563.

10. Fried $L P$, Tangen $C M$, Walston J, et al. Frailty in older adults: evidence for a phenotype. J Gerontol A Biol Sci Med Sci 2001;56(3):M146-M156.

11. Clegg A, Young J, lliffe S, Rikkert MO, Rockwood K. Frailty in elderly people. Lancet 2013;381(9868):752-762.

12. Espinoza SE, Quiben M, Hazuda HP. Distinguishing Comorbidity, Disability, and Frailty. Curr Geriatr Rep 2018;7(4):201-209.

13. Chen X, Mao G, Leng SX. Frailty syndrome: an overview. Clin Interv Aging 2014;9:433-441.

14. Angulo J, El Assar M, Álvarez-Bustos A, Rodríguez-Mañas L. Physical activity and exercise: Strategies to manage frailty. Redox Biol 2020;35:101513.

15. Dent E, Lien C, Lim WS, et al. The Asia-Pacific Clinical Practice Guidelines for the Management of Frailty. J Am Med Dir Assoc 2017;18(7):564-575.

16. Hendry A, Carriazo AM, Vanhecke E, Rodríguez-Laso Á; ADVANTAGE JA Work Package 7. Integrated Care: A Collaborative ADVANTAGE for Frailty. Int J Integr Care 2018;18(2):1.

17. Marcucci M, Damanti S, Germini F, et al. Interventions to prevent, delay or reverse frailty in older people: a journey towards clinical guidelines. BMC Med. 2019;17(1):193.

18. Roller-Wirnsberger R, Lindner S, Liew A, et al. European Collaborative and Interprofessional Capability Framework for Prevention and Management of Frailty-a consensus process supported by the Joint Action for Frailty
Prevention (ADVANTAGE) and the European Geriatric Medicine Society (EuGMS). Aging Clin Exp Res 2020;32(4):561-570.

19. Hoogendijk EO, Afilalo J, Ensrud KE, Kowal P, Onder G, Fried LP. Frailty: implications for clinical practice and public health. Lancet 2019;394(10206):1365-1375.

20. Guigoz Y, Vellas B, Garry PJ. Assessing the nutritional status of the elderly: The Mini Nutritional Assessment as part of the geriatric evaluation. Nutr Rev 1996;54(1 Pt 2):S59-S65.

21. Guralnik JM, Ferrucci L, Pieper CF, et al. Lower extremity function and subsequent disability: consistency across studies, predictive models, and value of gait speed alone compared with the short physical performance battery. J Gerontol A Biol Sci Med Sci 2000;55(4):M221-M231.

22. Rabin R, de Charro F. EQ-5D: a measure of health status from the EuroQol Group. Ann Med 2001;33(5):337-343.

23. Folstein MF, Folstein SE, McHugh PR. Mini-mental state. A practical method for grading the cognitive state of patients for the clinician. J Psychiatry Res 1975;12:189-198.

24. Dubois B, Touchon J, Portet F, et al. "Les 5 mots", épreuve simple et sensible pour le diagnostic de la maladie d'Alzheimer. Presse Med 2002:31:1696-1699.

25. Shulman Kl. Clock-drawing: is it the ideal cognitive screening test? Int J Geriatr Psychiatry 2000;15(6):548-61.

26. Malek-Ahmadi M, Small BJ, Raj A. The diagnostic value of controlled oral word association test-FAS and category fluency in single-domain amnestic mild cognitive impairment. Dement Geriatr Cogn Disord 2011;32(4):235-420.

27. Isaacs B, Kennie AT. The Set test as an aid to the detection of dementia in old people. Br J Psychiatry 1973;123(575):467-470.

28. Yesavage JA, Brink TL, Rose TL, et al. Development and validation of a geriatric depression screening scale: a preliminary report. J Psychiatr Res 1983:17(1):37-49.

29. Katz S. Assessing self-maintenance: activities of daily living, mobility, and instrumental activities of daily living. J Am Geriatr Soc 1983;31(12):721-727

30. Lawton MP, Brody EM. Assessment of older people: self-maintaining and instrumental activities of daily living. Gerontologist 1969;9(3): 179-186.

31. Hebert R, Carrier R, Bilodeau A. The Functional Autonomy Measurement System (SMAF): description and validation of an instrument for the measurement of handicaps. Age Ageing 1988;17:293-302.

32. Aguilova L, Sauzéon H, Balland É, Consel C, N'Kaoua B. AGGIR scale: a contribution to specifying the needs of disabled elders. Rev Neurol (Paris) 2014;170(3):216-221.

33. König HH, Heider D, Lehnert $\mathrm{T}$, et al. Health status of the advanced elderly in six European countries: results from a representative survey using EQ-5D and SF-12. Health Qual Life Outcomes 2010;8:143.

34. Tchalla AE, Dufour AB, Travison TG, Habtemariam D, Iloputaife I, Manor B, Lipsitz LA. Patterns, predictors, and outcomes of falls trajectories in older adults: the MOBILIZE Boston Study with 5 years of follow-up. PLoS One 2014;9(9):e106363.

35. Tchalla AE, Lachal F, Cardinaud N, Saulnier I, Bhalla D, Roquejoffre A, Rialle V, Preux PM, Dantoine T. Efficacy of simple home-based technologies combined with a monitoring assistive center in decreasing falls in a frail elderly population (results of the Esoppe study). Arch Gerontol Geriatr 2012;55(3):683-689.

36. Boyer S, Gayot C, Bimou C, Mergans T, Kajeu P, Castelli M, Dantoine T, Tchalla A. Prevalence of mild hyponatremia and its association with falls in older adults admitted to an emergency geriatric medicine unit (the MUPA unit). BMC Geriatr 2019:19(1):265.

37. Collard RM, Boter H, Schoevers RA, Oude Voshaar RC. Prevalence of frailty in community-dwelling older persons: a systematic review. J Am Geriatr Soc 2012;60(8):1487-1492.

38. Shamliyan T, Talley KM, Ramakrishnan R, Kane RL. Association of frailty with survival: a systematic literature review. Ageing Res Rev 2013;12(2):719-736.

39. Herr M, Arvieu JJ, Robine JM, Ankri J. Health, frailty and disability after ninety: Results of an observational study in France. Arch Gerontol Geriatr 2016;66:166-175

40. Vermeulen J, Neyens JC, van Rossum E, Spreeuwenberg MD, de Witte LP. Predicting ADL disability in community-dwelling elderly people using physical frailty indicators: a systematic review. BMC Geriatr 2011;11:33. 
41. Herr M, Arvieu JJ, Aegerter P, Robine JM, Ankri J. Unmet health care needs of older people: prevalence and predictors in a French cross-sectional survey. Eur J Public Health 2014;24(5):808-813.

42. Dubuc N, Hébert R, Desrosiers J, Buteau M, Trottier L. Disability-based classification system for older people in integrated long-term care services: the Iso-SMAF profiles. Arch Gerontol Geriatr 2006;42(2):191-206.

43. Treacy D, Hassett L. The Short Physical Performance Battery. J Physiother 2018;64(1):61.

44. Perracini MR, Mello M, de Oliveira Máximo R, Bilton TL, Ferriolli E, Lustosa $L P$, da Silva Alexandre T. Diagnostic Accuracy of the Short Physical Performance Battery for Detecting Frailty in Older People. Phys Ther 2020;100(1):90-98.

45. Makizako H, Shimada H, Doi T, et al. Physical frailty predicts incident depressive symptoms in elderly people: prospective findings from the Obu Study of Health Promotion for the Elderly. J Am Med Dir Assoc 2015;16(3):194-199.

46. Tucker KL. Nutrient intake, nutritional status, and cognitive function with aging. Ann N Y Acad Sci 2016;1367(1):38-49.

47. Verlaan S, Ligthart-Melis GC, Wijers SLJ, Cederholm T, Maier AB, de van der Schueren MAE. High prevalence of physical frailty among communitydwelling malnourished older adults-A systematic review and meta-analysis. J Am Med Dir Assoc 2017;18(5):374-382.

48. Khezrian M, Myint PK, McNeil C, Murray AD. A Review of Frailty Syndrome and Its Physical, Cognitive and Emotional Domains in the Elderly. Geriatrics (Basel). 2017;2(4):36

\section{Publisher's Note}

Springer Nature remains neutral with regard to jurisdictional claims in published maps and institutional affiliations.

Ready to submit your research? Choose BMC and benefit from:

- fast, convenient online submission

- thorough peer review by experienced researchers in your field

- rapid publication on acceptance

- support for research data, including large and complex data types

- gold Open Access which fosters wider collaboration and increased citations

- maximum visibility for your research: over $100 \mathrm{M}$ website views per year

At BMC, research is always in progress.

Learn more biomedcentral.com/submissions 